Baltic Astronomy, vol.2, 444, 1993.

WHOLE EARTH TELESCOPE DATA ON G 117-B15A AND G 226-29

\title{
S.O. Kepler
}

Instituto de Fisica, Universidade Federal do Rio Grande do Sul, 91501-970 Porto Alegre, RS - Brazil.

Received August 1, 1993.

Key words: methods: observational - techniques: photometric stars: white dwarfs - stars: oscillations - stars: individual: G 117B15A, G 226-29

\section{Abstract}

G 117-B15A was observed with the WET for $86.4 \mathrm{~h}$ in March 1990 (XCOV4) and $9.1 \mathrm{~h}$ in March 1993 (XCOV9). The most recent result for the rate of period change for the $215 \mathrm{~s}$ pulsation mode is $\dot{P}=(3.2 \pm 2.8) \times 10^{-15} \mathrm{~s} / \mathrm{s}$, which includes the March 1993 data. We have taken Fourier spectra of all the data on G 117-B15A available since 1974. Besides the six multiplets known before (see Kepler et al. 1982, ApJ, 254, 676), we have found a few other peaks with probability of being due to noise smaller than 1 in 1000: $79.5 \mathrm{~s}$, amp $=0.4 \mathrm{mma}$, frequency $=2 f_{1}+f_{2}$ (here $f_{1}$ is the frequency of the $215 \mathrm{~s}$ peak and $f_{2}$ is the frequency of the $304 \mathrm{~s}$ peak) and at $736.6 \mathrm{~s}, \mathrm{amp}=0.9 \mathrm{mma}$, frequency $=f_{1}-f_{2}$.

For the six multiplets known before, we find that all, but perhaps the $215 \mathrm{~s}$ mode, are split into several components, but not simple multiplets. The low duty cycle of the WET campaign on G 117-B15A has introduced severe window effects, which, coupled with the complexity of the observed multiplets, require more WET observations to untangle.

G 226-29 was observed for $121 \mathrm{~h}$ in February 1992 (XCOV7), and we only detected the three previously known frequencies which are close to $109 \mathrm{~s}$, down to $0.5 \mathrm{mma}$ (see Fig. 1, this journal, p. 518). The results, together with an explanation of the False Alarm Probability on our FTs, will be published elsewhere. 